Departement für Pferde, Klinik für Pferdemedizin der Vetsuisse-Fakultät Universität Zürich

Direktor: Prof. Dr. med. vet. Colin C. Schwarzwald

Arbeit unter wissenschaftlicher Betreuung von

Prof. Dr. med. vet. Colin C. Schwarzwald

\title{
Assessment of systolic and diastolic function in clinically healthy horses using ambulatory acoustic cardiography
}

\section{Inaugural-Dissertation}

zur Erlangung der Doktorwürde der

Vetsuisse-Fakultät Universität Zürich

vorgelegt von

Nicole Zuber

von Othmarsingen AG, Matzendorf SO

genehmigt auf Antrag von

Prof. Dr. med. vet. Colin C. Schwarzwald, Referent 


\section{Table of contents:}

Abstract English $\quad$ Page 4

Abstract German $\quad$ Page 5

$\begin{array}{ll}\text { Summary } & \text { Page } 6\end{array}$

Introduction $\quad$ Page 6

$\begin{array}{ll}\text { Materials and methods } & \text { Page } 7\end{array}$

$\begin{array}{ll}\text { Study design } & \text { Page } 7\end{array}$

$\begin{array}{ll}\text { Study group } & \text { Page } 7\end{array}$

$\begin{array}{ll}\text { Data recordings } & \text { Page } 7\end{array}$

$\begin{array}{ll}\text { Data processing and analyses } & \text { Page } 8\end{array}$

$\begin{array}{ll}\text { Statistical analysis } & \text { Page } 9\end{array}$

Results

Page 9

Discussion $\quad$ Page 9

$\begin{array}{ll}\text { Conclusion } & \text { Page } 13\end{array}$

Authors' declaration of interests $\quad$ Page 13

Ethical animal research $\quad$ Page 13

$\begin{array}{ll}\text { Sources of funding } & \text { Page } 13\end{array}$

Acknowledgements $\quad$ Page 13

$\begin{array}{ll}\text { Authorship } & \text { Page } 13\end{array}$

$\begin{array}{ll}\text { Manufacturers' addresses } & \text { Page } 13\end{array}$

$\begin{array}{ll}\text { References } & \text { Page } 13\end{array}$

$\begin{array}{ll}\text { Supporting Information } & \text { Page } 15\end{array}$ 


\section{Summary}

The objective of this study was to establish the use of an acoustic ECG monitor (Audicor) in healthy horses. It provides noninvasive, examiner-independent, continuous analysis combining ECG and phonocardiography to calculate indices of cardiac mechanical activity and haemodynamics. Device usability was investigated, reference intervals calculated and reproducibility of analyses assessed. Methods: Continuous overnight recordings were obtained in 123 healthy horses. Electromechanical activating time (EMAT), rate-corrected EMATc, left ventricular systolic time (LVST), rate-corrected LVSTc and intensity and persistence of the third and fourth heart sound (S3, S4) were reported. Associations with age and reproducibility of analyses were assessed. Results: Audicor recordings of diagnostic quality were obtained, with an artefact-free recording time of 1:08-14:03h. $44.8 \%$ of the horses had atrial premature complexes. $4.3 \%$ had ventricular premature complexes. Reference intervals were reported. S3 was significantly more often graded $\geq 5$ (scale $0-10$ ) in younger compared to older horses. The between-day coefficient of variation ranged from 2.5 to $7.7 \%$ for EMAT, EMATc, LVST and LVSTc. Conclusions: Overnight Audicor recordings are feasible in horses. Combining ambulatory ECG and phonocardiography allows noninvasive, continuous assessment of variables representing systolic and diastolic cardiac function.

Keywords: horse; electrocardiogram; phonocardiogram; heart sounds; cardiovascular 


\section{Zusammenfassung}

Ziel der Studie war es, die Anwendung des Audicor Gerätes durch Bestimmung von Referenzwerten und Ermitteln der Reproduzierbarkeit im klinischen Alltag zu etablieren. Das Audicor Gerät vereint kontinuierliches EKG und Phonokardiogramm, ist nicht-invasiv und Untersucher-unabhängig. Aussagen über die mechanische und hämodynamische Aktivität des Herzens werden so ermöglicht. Methode: An 123 gesunden Pferden wurden Aufnahmen über Nacht angefertigt. Die festgehaltenen Variablen waren u.a. elektromechanische Aktivierungszeit (EMAT), Frequenzkorrigierte EMATc, linksventrikuläre Systolenzeit (LVST), Frequenz-korrigierte LVSTc, Intensität und Persistenz des 3. und 4. Herztones (S3, S4). Die Altersabhängigkeit und die Reproduzierbarkeit der Variablen wurden untersucht. Resultate: Aufnahmen mit artefaktfreier Zeit von 1:08-14:03h wurden angefertigt. 44.8\% der Pferde zeigten supraventrikuläre Extrasystolen, $4.3 \%$ zeigten ventrikuläre Extrasystolen. Referenzintervalle für alle Variablen wurden berechnet. Bei jungen Pferden wurden signifikant mehr S3 $\geq 5$ (Skala 0-10) registriert. Der Variationskoeffizient für Aufnahmen an verschiedenen Tagen variierte zwischen 2.5 und 7.7\% für EMAT, EMATc, LVST und LVSTc. Fazit: Aufnahmen mit Audicor beim Pferd sind nicht-invasiv durchführbar und erlauben kontinuierliche Aussagen über die systolische und diastolische Herzfunktion. Schlüsselwörter: Pferd; Elektrokardiogramm; Phonokardiogramm; Herztöne; Kardiovaskulär. 


\title{
Assessment of systolic and diastolic function in clinically healthy horses using ambulatory acoustic cardiography
}

\author{
N. ZUBER ${ }^{\dagger}$, M. ZUBER and C. C. SCHWARZWALD ${ }^{\dagger}, \star$ \\ ${ }^{\dagger}$ Clinic for Equine Internal Medicine, Vetsuisse Faculty, University of Zurich, Zurich, Switzerland \\ *Division of Cardiology, University of Zurich, Zurich, Switzerland. \\ *Correspondence email: cschwarzwald@vetclinics.uzh.ch; Received: 09.12.17; Accepted: 21.08.18
}

\section{Summary}

Background: Assessment of cardiac electromechanical function in horses requires training, experience and specialised equipment and does not allow continuous monitoring over time.

Objectives: The objective of this study was to establish the use of an acoustic ECG monitor (Audicor ${ }^{\circledR}$ ) in healthy horses. It provides noninvasive, examiner-independent, continuous analyses combining ECG and phonocardiography to calculate indices of cardiac mechanical activity and haemodynamics. Device usability was investigated, reference intervals calculated and reproducibility of analyses assessed.

Study design: Prospective descriptive study.

Methods: Continuous overnight recordings were obtained in 123 healthy horses. ECG and acoustic cardiography analyses were performed. Electromechanical activating time (EMAT), rate-corrected EMATc, left ventricular systolic time (LVST), rate-corrected LVSTc and intensity and persistence of the third and fourth heart sound $(\mathrm{S3}, \mathrm{S4})$ were reported. Associations with age and reproducibility of analyses were assessed.

Results: Audicor ${ }^{\circledR}$ recordings of diagnostic quality were obtained in 116 horses, with an artefact-free recording time of 1:08-14:03 h (mean 10:21 h). $44.8 \%$ of the horses had atrial premature complexes (up to $0.18 \%$ of analysed beats), $4.3 \%$ had ventricular premature complexes (up to $0.021 \%$ of analysed beats). Reference intervals for acoustic cardiography variables were reported. S3 was significantly more often graded $\geq 5$ (scale 0-10) in younger compared to older horses $\left(P=0.0036, R^{2}=0.072\right.$ ). The between-day coefficient of variation ranged from 2.5 to $7.7 \%$ for EMAT, EMATC, LVST and LVSTC.

Main limitations: Audicor ${ }^{\circledR}$ algorithms are based on human databases. Horses were deemed clinically healthy without advanced diagnostics. Some data were lost because of technical difficulties, artefacts and noises.

Conclusions: Overnight Audicor ${ }^{\circledR}$ recordings are feasible in horses. Combining ambulatory ECG and phonocardiography allows noninvasive, continuous assessment of variables representing systolic and diastolic cardiac function. ECG rhythm analyses require over-reading by a specialist, but acoustic cardiography variables are based on automated algorithms independent of examiner input. Further studies are required to establish the clinical value of acoustic cardiography in horses.

Keywords: horse; electrocardiogram; phonocardiogram; heart sounds; cardiovascular

\section{Introduction}

Horses are flight animals and high-level athletes that depend on an efficient and powerful cardiovascular system to manage the physical requirements of stress and rapid locomotion. Heart diseases are common in horses and - if severe enough - lead to exercise intolerance and poor performance [1]. Cardiovascular disease accounts for $5.1 \%$ of deaths in 15-23 years old horses and for $8.5 \%$ of deaths in horses older than 24 years [2]. The most common cardiac diseases include mitral and aortic insufficiency [2-5], ventricular septal defects [5,6] and arrhythmias such as atrial fibrillation $[5,7,8]$. Because progression of disease into heart failure is relatively rare and heart failure is only diagnosed when overt clinical signs of exercise intolerance and congestion develop, its real prevalence is largely unknown [5].

A variety of different diagnostic methods are routinely available to assess the cardiovascular system in horses. In field practice, these primarily include physical examination, careful cardiac auscultation, exercise testing and laboratory examination. Specialised clinicians and larger referral centres also offer resting electrocardiography (ECG), stress ECG, ambulatory Holter ECG and event monitoring, transthoracic echocardiography, noninvasive blood pressure measurement and cardiac catheterisation [5]. These more advanced methods require extensive training and experience and access to the necessary equipment. Hence, these methods are not readily available in field practice. Diagnostic findings are often difficult to interpret and are subject to operator error and intra- and interobserver variability [9-15].

The Audicor $\AA$ technology represents a noninvasive, ambulatory, wireless diagnostic point-of-care device that can be applied by any clinician even in remote field settings. The device has been developed for use in people with heart disease. It continuously records a singlelead surface electrocardiogram combined with a phonocardiogram [16]. Using fully automated ECG signal and heart sound analyses, focusing on systolic time intervals and diastolic heart sound quantification, it provides a comprehensive, continuous assessment of the electrical and haemodynamic characteristics of the heart of human patients for up to several days duration [17-21]. In particular, the device is used in cardiac patients for diagnostic purposes [19,22-25], to monitor and optimise therapy [26], to predict prognosis of heart disease [20] and to screen large populations of patients [16]. Among other variables, Audicor $^{\oplus}$ measures the electromechanical activation time (EMAT) as the time interval between the onset of the electrocardiographic Q wave and the first heart sound. Furthermore, it can quantify the strength of the third heart sound (S3) and provides a compound variable called systolic dysfunction index (SDI) that includes both EMAT and strength of S3 (see Fig 1 and Supplementary Item 1). In people, strong correlations were found between the strength of S3 and left ventricular (LV) end-diastolic filling pressures and between EMAT and the maximum rate of systolic LV pressure change ( $\left.L V \quad d p / d t_{\max }\right)$ and LV ejection fraction, respectively. Consequently, EMAT, S3 and SDI were shown to be highly specific to diagnose LV dysfunction and heart failure $[16,27]$

In a recent study, it was shown that systolic time intervals can be altered in horses with mitral and aortic insufficiency, but their clinical value could not be well documented and remains uncertain at this time [28]. Similarly, S3 can be heard in healthy horses and in horses with heart disease, but lacking accurate methods for quantification of the strength of S3, its 


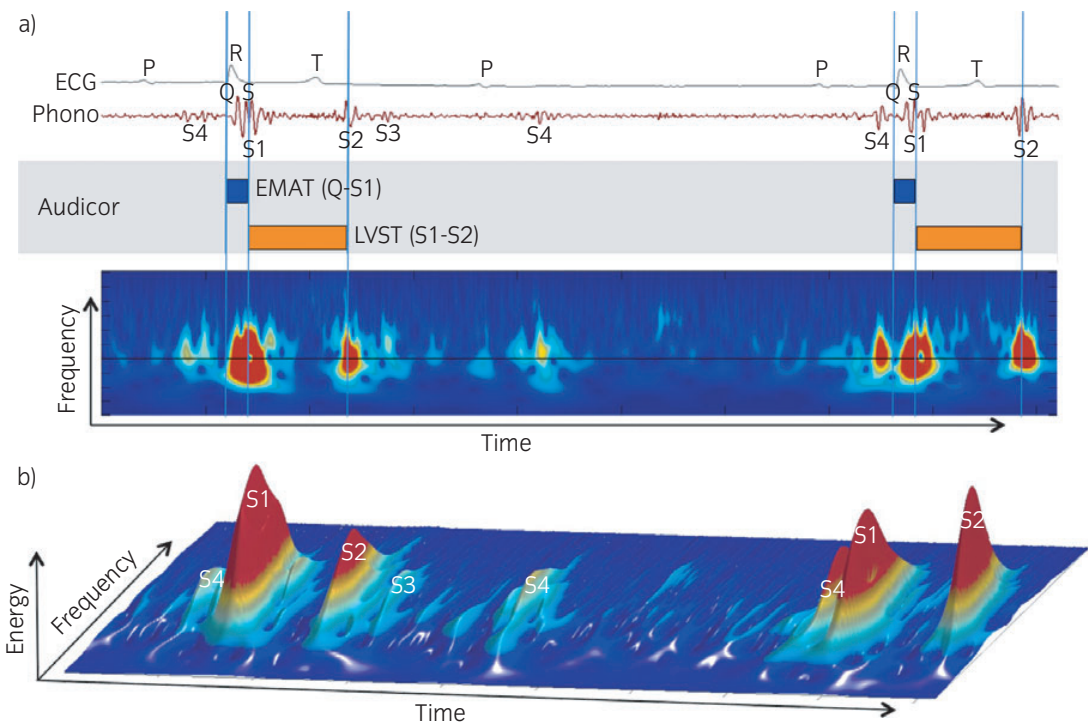

Fig 1: a) Electrocardiogram and phonocardiogram recorded by the Audicor ${ }^{\circledR}$ Dx Patch device, showing a normal sinus rhythm with a second-degree AV block. The timing of the measurements (onset of QRS complex, S1, S2) is indicated by vertical lines. The two-dimensional representation of the phonocardiogram displays the sound frequency on the $y$-axis, whereas the acoustic energy is colour-coded (low-energy signals in blue, intermediate-energy signals in yellow, high-energy signals in red). Note that isolated fourth heart sounds, such as in second-degree AV block, are neither detected nor quantified by the analysis algorithm. EMAT, Electromechanical activation time; LVST, Left ventricular systolic time; S1, first heart sound; S2, second heart sound; S3, third heart sound; S4, fourth heart sound. b) three-dimensional illustration of the phonocardiogram, showing the four heart sounds (S1-S4) characterised by timing, sound frequency and acoustic energy. Note that the 3D phonocardiogram has a lower temporal resolution and is created by Audicor ${ }^{\circledR}$ for illustration only.

diagnostic and prognostic value in horses with heart disease is not well established [5,29].

We suggest that Audicor ${ }^{\circledR}$ could be useful for diagnosis and staging of cardiac diseases in horses, providing insights into rhythm disturbances and haemodynamic abnormalities through simultaneous assessment of the electrical and mechanical activity of the heart. This could be helpful to understand the true prevalence of clinically relevant heart disease and improve medical care of horses.

In a previous pilot study, the Audicor ${ }^{\circledR}$ device has been shown to be able to record physiologic data of sufficient quality to be analysed and to derive a variety of cardiovascular variables in horses (N. Zuber, unpublished data). The aim of this study was to collect acoustic cardiography data in a larger group of clinically healthy horses to measure systolic time intervals, to quantify the prevalence and intensity of diastolic heart sounds, to investigate their age-related changes and to assess reference intervals and test-retest variability in acoustic cardiography variables.

\section{Materials and methods}

\section{Study design}

The study was designed as a prospective observational trial.

\section{Study group}

Clinically healthy horses were recruited from horse stables in various regions of Switzerland. A total of 123 horses $(113$ Warmbloods, 4 Thoroughbreds, 6 Standardbreds) between 3 and 27 years of age (11 \pm 5 [mean \pm s.d.]) were included in the study. The group contained 64 mares, 56 geldings and 3 stallions with a height at the withers of $168 \pm 6 \mathrm{~cm}$ and a body weight of $546 \pm 53 \mathrm{~kg}$. It consisted of pleasure horses and sports horses used at different levels of showjumping, dressage and 3-day eventing

The horses were deemed clinically healthy based on a medical and performance history and a comprehensive physical examination (including careful examination of mucous membranes, jugular veins, peripheral pulses and auscultation of heart and lungs). Horses that suffered from cardiorespiratory diseases, other medical issues or any painful conditions were not included in the study. All horses had to stay free of food supplements or drugs containing adrenergic or anticholinergic agents, vasodilators, diuretics, ACE inhibitors, cardiac glycosides or antiarrhythmics for 1 month prior to the recordings. In addition, no analgesics or antiinflammatory drugs were allowed 1 week prior to the recording.

\section{Data recordings}

Data recordings were obtained by a single operator (NZ) with the Audicor ${ }^{\circledR}$ Dx Patch device ${ }^{a}$ (Fig 2a). This $10 \mathrm{~cm}$ long, battery-driven device contains two dry electrodes for recording of the digital surface ECG signal and a high-fidelity accelerometer for heart sound recordings and for assessment of body position, physical activity and respiratory effort (body position, activity levels and respiratory effort were not analysed within the scope of this study). Internal digital storage capabilities allow for up to $24 \mathrm{~h}$ of data recording at a sampling rate of $500 \mathrm{~Hz}$.

For this study, the Dx Patch device was fixed with a modified surcingle to the left side of the horses' thorax (Fig 2b). The device was positioned between the 5 and 7th intercostal space, in vertical orientation, with the white part of the device at the level of the olecranon and the green part (containing the accelerometer) directed dorsally. Due to variations in body conformation (thorax, withers), the surcingle could not be placed in a completely standardised fashion. Furthermore, the position of the sound sensor could not be optimised for the varying point of maximum intensity of the heart sounds in individual horses. Nonetheless, this location allowed recording of a modified base-apex ECG lead and a phonocardiogram of adequate quality (N. Zuber, unpublished data; see Fig 1).

All recordings were performed in summer and early fall and the length of the horses' hair coat varied. Adequate skin contact was ensured using dry ECG electrodes with a $\mathrm{Ag} / \mathrm{AgCl}$ core $^{\text {b }}$ providing a stable and low-noise interface between skin and device. At the beginning of the recordings, the fur was slightly wetted with water to allow immediate electrical contact. Once the mounting system was in place, slight sweating occurring underneath the mounting system resulted in sufficient electrical contact for the duration of the recordings. Neither clipping nor shaving was required. 
a)

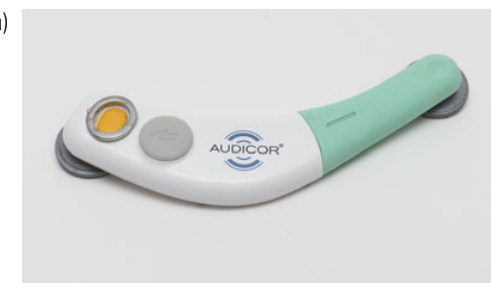

b)

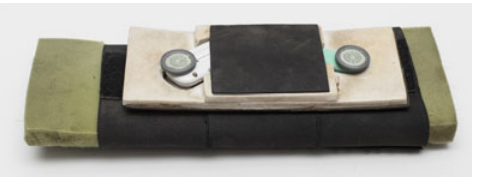

c)

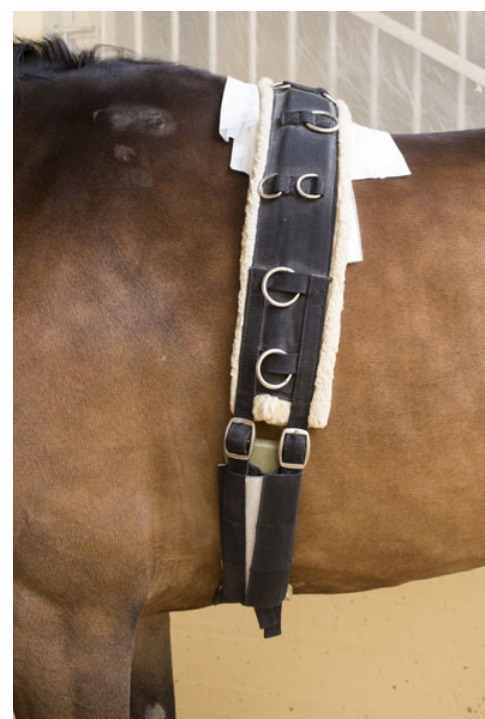

Fig 2: a) Audicor ${ }^{\circledast}$ Dx Patch device, b) Audicor ${ }^{\boxplus}$ Dx Patch device with attachment. c) Illustration of the experimental set-up on the horses.

After instrumentation, the device was connected via a proprietary Wi-Fitype low-power wireless connection to a laptop computer and the recording was started. Successful operation of the device and adequate quality of recordings were immediately verified by visual inspection of the ECG and phonocardiogram on a 10-s snapshot recording (Fig 1). The quality of the ECG was considered sufficient if the baseline was stable; $P$, QRS and T waves were clearly discernible; and the ECG tracing was not disturbed by excessive motion artefacts. Once proper device function was verified, the wireless connection was discontinued and the device continued recording to its internal data memory.

The recordings were conducted in a standardised way, starting between 17:00 and 20:00 and ending between 05:00 and 08:00 the following day, aiming to obtain recordings of at least $10 \mathrm{~h}$ duration. Overnight recordings were chosen to reduce the influence of environmental factors and individual daytime activity patters, with the aim to improve data quality, allow better standardisation of recordings and facilitate comparison of results between horses. All recordings were taken in the horses' familiar environment and the owners were advised to leave the horses undisturbed until recordings were finished. No other restrictions were imposed and barn work continued as usual. After completion of recordings, the raw data files were copied via USB connection from the Dx Patch device onto a laptop computer

In a subset of 10 randomly selected horses, recordings were repeated every second day to obtain a total of three recordings per horse, allowing assessment of the reproducibility of acoustic cardiography variables in this group of horses.

\section{Data processing and analyses}

Copies of all data files were renamed using a coding system. Blinded data processing and analyses were subsequently performed by Inovise Medical Inc. $^{\text {C }}$ using proprietary data analysis software (CA 300). The software algorithm relies on a sophisticated wavelet-based signal processing technique to evaluate heart sound data in relation to the ECG data (Fig 1) [16]. The diagnostic algorithm has been trained to set the sound fiducial points based on large (human) datasets. The time-frequency analysis allows separation of heart sounds from murmurs, external noise, pulmonary sounds and artefacts. Each sound component has a very specific time-frequency "fingerprint", so that all four heart sounds can be identified and timed. In contrast to auscultation and traditional phonocardiography, the sound energy of the third and fourth heart sound (S3 and S4) is quantified, so that S3 and S4 are recorded as continuous rather than dichotomous variables. Sinus arrhythmia does not significantly impact analyses, because all indices are calculated as an average of all beats over $10 \mathrm{~s}$. However, an isolated S4, such as in second-degree AV block, is neither detected nor quantified by the analysis algorithm, because it lacks association with a QRS-T complex. The algorithm also does not analyse for $\mathrm{S} 4$ in the case of atrial arrhythmia with AV blocks, atrial fibrillation or atrial flutter. Comparison of the filtered signals from dogs, pigs, horses and other animals to those of humans does not show any fundamental differences concerning their frequency content and the placement of the relevant fiducial points (Inovise Medical Inc., personal communication).

The analyses provided by Inovise Medical Inc. included a Holter ECG report, a "cardiac findings" report and a 10-s "baseline snapshot" report. Such "snapshots" are routinely recorded in people to obtain a point-of-care set of variables for assessment of LV systolic and diastolic function without the necessity of an ambulatory long-term recording [30]. Artefact periods were detected based on an automated proprietary algorithm. Segments were labelled as artefacts if the ECG signal did not allow consistent beat detection or if the frequency domain of the sound signal had a low signalto-noise ratio. All labelled artefact periods were confirmed by a trained technician and subsequently excluded from analyses.

Holter ECG analyses were performed on all complete recordings based on the entire recording time available. The report included the duration of analysed time, the duration of artefact-free time, and the number of analysed beats; the minimum, maximum and average heart rate; and the number and type of supraventricular and ventricular arrhythmias. The rhythm diagnoses were confirmed and if necessary corrected by a trained ECG technician. All detected rhythm events were additionally verified by an equine clinician with expertise in rhythmology (CS).

Automated acoustic cardiography analysis was performed in all subjects during a fixed time window from 22:00 to 05:00, resulting in a maximum analysis period of $7 \mathrm{~h}$ for all horses. Artefact periods were excluded from analyses. At least $4 \mathrm{~h}$ of ECG and phonocardiogram recordings were required for successful acoustic cardiography analyses. The following variables were generated and averaged over the entire analysed time window and reported as "cardiac findings" (see Fig 1 and Supplementary Item 1): Heart rate (HR), QRS duration (QRS), heart rate-corrected QT interval (OTC), electromechanical activation time (EMAT), heart ratecorrected electromechanical activation time (EMATC), left ventricular systolic time (LVST) and the heart rate-corrected left ventricular systolic time (LVSTC). Furthermore, the "cardiac findings" report contained the percentage of 10-s segments contained in the analysis period with EMATC $\geq 15 \%$; the strength (as a function of intensity and persistence) of the third (S3) and fourth heart sound (S4) $\geq 5$; and a systolic dysfunction index (SDI, as a function of QRS duration, QT interval, EMATC, and S3) $\geq 5$ and $\geq 7.5$, respectively. Note that in humans, S3 and S4 are considered to be present if their power is $>5$ (on a scale of $0-10$ ) [31] and EMATC $>15 \%, \mathrm{SDI}>5$ and $\mathrm{SDI} \geq 7.5$ were found to predict LV systolic dysfunction and poor prognosis $[20,23,30,32]$. Hence, the respective cut-off values used by the analysis software were those established by the manufacturer and approved by the FDA to guide diagnosis in human patients. It was not possible to adjust these cut-offs for the purpose of this study.

A 10-s "baseline snapshot" analysis was created from data recorded during the first 2 min after start of the recording. The variables included in 
the snapshot analysis were the following: HR, EMAT, EMATC, LVST, LVSTC S3, S4 and SDI (see Supplementary Item 1 for a glossary of all variables).

\section{Statistical analysis}

Data analysis and statistics were performed using commercially available software (Microsoft Excel for Mac 2011, v14.6.4 ; GraphPad PRISM for Mac OS X, v7.0be)

Summary statistics (mean, s.d., median) and reference intervals of each variable were calculated using dedicated software (Reference Value Advisor $\mathrm{v} 2.1^{\mathrm{f}}$ ). Due to the large sample size, nonparametric reference intervals were calculated. The $90 \%$ confidence intervals (Cls) of the limits of the reference intervals were determined using a bootstrap method. The relationship of obtained variables to age, height and body weight was assessed using linear regression analyses. For non-normally distributed variables, linear regression analyses were conducted on log-transformed data. Validity of the linear model was assessed by graphical display of the data and assessment of normal probability plots of the residuals. Bonferroni correction was performed to adjust for the family-wise error rate after conducting eight individual linear regression analyses in the "snapshot" statistics (adjusted level of significance, $P=0.05 / 8=0.0063$ ) and 12 individual linear regression analyses in the "cardiac findings" statistics (adjusted level of significance, $\mathrm{P}=0.05 / 12=0.0042$ )

The horses were then divided into five age groups $(0-5,6-10,11-15$, 16-20, 21-27 years) and groups were compared using one-way ANOVA followed by Tukey post hoc test (for normally distributed variables) or Kruskal-Wallis test followed by Dunn's post hoc test (for non-normally distributed variables). To assess the data for differences in sex, only mares and geldings were considered, while stallions were excluded because of insufficient group size $(n=3)$ for statistical analyses. Sex groups were compared using unpaired $t$ test (for normally distributed variables) or Mann-Whitney test (for non-normally distributed variables). Normal distribution and equal variance of the data in the different groups were assessed by graphical display of the data and inspection of distribution and normal probability plots of the residuals.

The test reproducibility was quantified based on the three repeated measurements obtained in each of the 10 randomly selected horses. Reproducibility was quantified by use of the within-subject variance for repeated measurements (residual mean square) determined by a one-way ANOVA with horses as the groups. The within-subject standard deviation $\left(s_{w}\right)$ was calculated as the square root of the residual mean square. Measurement variability and recording variability were reported in two ways. First, for all normally distributed variables, the within-subject CV expressed as a per cent value was calculated as $C V=s_{w} /$ mean $\times 100$ The CV primarily served to compare the degree of variability between different variables and across different studies. However, the interpretation of the $\mathrm{CV}$ is difficult and not standardised and its clinical applicability is limited [33]. Therefore, in addition to the CV, the absolute value below which the difference between two measurements will lie with 95\% probability was estimated for all variables following the British Standards Institution (BSI) recommendations as follows: $1.96 \times \sqrt{2} \times \mathrm{s}_{w}=2.77 \times \mathrm{s}_{w}$ [34]. This measure of variability considers uncertainty of the point estimates and uncertainty of prediction associated with repeated measurements [33]. When applied clinically, the magnitude of change observed in a variable over time can be put in relation to the BSI value, to judge whether the change might be simply due to measurement error and physiologic variability (observed change $\leq \mathrm{BSI}$ value) or whether the change might represent a true change over time (observed change $>$ BSI value)

\section{Results}

Acoustic cardiography "snapshot" and ambulatory ECG recordings could be obtained in all 123 horses included in the study and all horses were in sinus rhythm. However, in five recordings, the device had turned off inadvertently during data collection, and in two horses, it detached from the surcingle, resulting in insufficient data for Holter analyses and "cardiac findings" reports in those seven cases (5.7\% of horses). In additional 19 cases, the surcingle dislodged from its designated position during the recording, and the ECG signal was temporarily lost due to a loosened contact of the dry electrode to the skin. Nonetheless, all those 19 recordings contained enough data to perform all intended analyses. Hence, data from 116 horses were included in the Holter and "cardiac findings" statistics.

For complete Holter ECG analyses $(n=116)$, the mean duration of analysed time was 12:22 $\mathrm{h}$ (s.d. 0:51 h, range 10:14-14:36 h) and the mean artefact-free time was 10:21 h (s.d. 2:44 h, range 1:08-14:03 h), including a mean of 22,813 beats (s.d. 6070, range 3042-35,392 beats). The average heart rate was 37 beats/min (mean; s.d. 4 beats/min) and the minimum and maximum heart rates were 30 beats/min (s.d. 4 beats $/ \mathrm{min}$ ) and 91 beats/min (s.d. 24 beats/min), respectively. During manual verification of the automated rhythm diagnoses, 1260 complexes that were labelled as atrial premature complexes (APC) and seven complexes that were labelled as ventricular premature complexes (VPC) were reclassified as normal beats; five beats that were labelled as APCs were reclassified as VPCS; and one beat that was labelled as normal beat was reclassified as APC. Fifty-two of 116 horses (44.8\%) had between one and 27 APCs, corresponding to a minimum of $0.0032 \%$ and a maximum of $0.18 \%$ of all analysed beats. Five of 116 horses (4.3\%) had between one and four VPCS, corresponding to a minimum of $0.0034 \%$ and a maximum of $0.021 \%$ of all analysed beats.

Summary statistics of the "cardiac findings", their reference intervals and their relationship to age, body weight and height are shown in Table 1. The S3 $(\geq 5)$ and S4 ( $\geq 5)$ were significantly but weakly related to age; EMATC, LVST and EMATC $(\geq 15 \%)$ were significantly related to body weight; and S3 $(\geq 5)$ was significantly related to height. However, $R^{2}$ was low for all relationships. After Bonferroni correction to adjust for the family-wise error rate, only the percentage detected 10-s segments in which the power of the third heart sound was $\geq 5$ (S3 $[\geq 5]$ ) remained significantly but weakly related to age, while none of the other variables was correlated with age, body weight or height. Comparisons of variables between age groups are summarised in Fig 3 and Supplementary Item 2. Only S3 ( $\geq 5)$ was significantly different between the age groups $0-5$ and 16-20 years $(P=0.027)$. Comparisons between sex groups revealed that $Q R S$ duration was significantly shorter in mares $(114 \pm 10 \mathrm{~ms}$, 95-130 ms [mean \pm s.d., 5-95th percentile]) compared to geldings $(120 \pm 21 \mathrm{~ms}, 106-133 \mathrm{~ms}$; $\mathrm{P}=0.037$, difference between means $6 \mathrm{~ms}, 95 \% \mathrm{Cl}$ of difference of means 0.4-13 ms), while none of the other variables was significantly different between groups.

Summary statistics of "snapshot" analyses and their relationship to age, body weight and height are shown in Table 2. Heart rate and LVST were significantly related to age; S3 strength was significantly related to body weight; and none of the "snapshot" variables was significantly related to height. After Bonferroni correction to adjust for the family-wise error rate, only $\mathrm{HR}_{\text {bas }}$ was significantly but weakly related to age. Comparisons between sex groups revealed that $\mathrm{SDI}_{\text {bas }}$ was significantly lower in mares $(2.3 \pm 1.0,1.1-4.4)$ compared to geldings $(2.8 \pm 1.0,1.4-5.0 ; P=0.024$, difference between means $0.5,95 \% \mathrm{Cl}$ of difference of means $0.06-0.8$ ), while none of the other variables was significantly different between groups.

The reproducibility of "cardiac findings" and "snapshot" variables is summarised in Table 3. Overall, variability in normally distributed "cardiac findings" variables, quantified using the between-day CV, was considerably lower than variability in the baseline "snapshot" variables. The coefficient of variation was not calculated for non-normally distributed "cardiac findings" variables including EMATc $(\geq 15 \%)$, S3 ( $\geq 5)$, S4 ( $\geq 5)$, SDI $(\geq 5)$ and SDI $(\geq 7.5)$. However, based on their BSI value, relative to their median and range, these variables showed moderate to high variability.

\section{Discussion}

This is the first study describing the feasibility, reliability and reference intervals of acoustic cardiography variables in horses. The ambulatory data included single-lead ECG recordings that were used for conventional Holter analyses. Furthermore, the technology allowed continuous recording of haemodynamic systolic and diastolic data by combined, simultaneous analysis of ECG and heart sounds throughout the recording period.

Ambulatory Holter ECG recordings have previously been used in horses for heart rate and rhythm analyses over prolonged periods of time [5]. 
TABLE 1: Summary statistics and reference intervals of "cardiac findings" variables obtained in clinically healthy horses ( $\mathrm{n}=116$ ).

\begin{tabular}{|c|c|c|c|c|c|c|c|c|c|c|c|c|}
\hline \multirow[b]{3}{*}{ Variable } & \multirow[b]{3}{*}{ Unit } & \multicolumn{5}{|c|}{ Summary statistics } & \multicolumn{6}{|c|}{ Linear regression } \\
\hline & & \multirow[b]{2}{*}{ Mean } & \multirow[b]{2}{*}{ s.d. } & \multirow[b]{2}{*}{ Median } & \multirow{2}{*}{$\begin{array}{l}\text { Lower limit of } \\
\text { reference } \\
\text { interval }(90 \% \mathrm{Cl})\end{array}$} & \multirow{2}{*}{$\begin{array}{l}\text { Upper limit of reference } \\
\text { interval }(90 \% \mathrm{Cl})\end{array}$} & \multicolumn{2}{|l|}{ Age } & \multicolumn{2}{|c|}{ Body weight } & \multicolumn{2}{|l|}{ Height } \\
\hline & & & & & & & $\mathrm{R}^{2}$ & $P$ & $\mathrm{R}^{2}$ & $P$ & $\mathrm{R}^{2}$ & $P$ \\
\hline $\mathrm{HR}$ & beats/min & 36 & 3.7 & 36 & $28(25-30)$ & $43(41-44)$ & 0.021 & 0.12 & $<0.01$ & 0.89 & $<0.01$ & 0.80 \\
\hline QRS & $\mathrm{ms}$ & 116 & 9.4 & 116 & 95 (92-99) & $132(131-140)$ & $<0.01$ & 0.56 & 0.032 & 0.055 & $<0.01$ & 0.77 \\
\hline QTC & $\mathrm{ms}$ & 385 & 25.2 & 385 & 338 (319-344) & 439 (426-455) & $<0.01$ & 0.47 & $<0.01$ & 0.87 & 0.011 & 0.25 \\
\hline EMAT & $\mathrm{ms}$ & 108 & 15.4 & 107 & $78(61-86)$ & $142(134-153)$ & 0.020 & 0.13 & $<0.01$ & 0.67 & $<0.01$ & 0.38 \\
\hline EMATC & $\%$ & 7.5 & 2.5 & 7 & $4.0(4.0-5.0)$ & $14.1(13.0-16.0)$ & 0.011 & 0.26 & 0.039 & 0.034 & $<0.01$ & 0.90 \\
\hline LVST & $\mathrm{ms}$ & 468 & 60.4 & 483 & 278 (238-305) & $533(524-538)$ & $<0.01$ & 0.96 & 0.047 & 0.020 & 0.023 & 0.11 \\
\hline LVSTC & $\%$ & 29.3 & 2.4 & 29 & $23.9(21.0-25.0)$ & $34.0(33.0-34.0)$ & $<0.01$ & 0.97 & $<0.01$ & 0.44 & 0.020 & 0.13 \\
\hline EMATC $(\geq 15 \%)$ & $\%$ & $\mathrm{n} / \mathrm{a}$ & $\mathrm{n} / \mathrm{a}$ & 3.5 & $0.0(0.0-0.2)$ & $49.3(37.5-73.4)$ & 0.033 & 0.054 & 0.043 & 0.028 & $<0.01$ & 0.45 \\
\hline S3 $(\geq 5)$ & $\%$ & $\mathrm{n} / \mathrm{a}$ & $\mathrm{n} / \mathrm{a}$ & 1.9 & $0.2(0.1-0.4)$ & $19.7(13.5-23.4)$ & 0.072 & $0.0036^{*}$ & 0.011 & 0.27 & 0.045 & 0.023 \\
\hline$S 4(\geq 5)$ & $\%$ & $\mathrm{n} / \mathrm{a}$ & $\mathrm{n} / \mathrm{a}$ & 1.7 & $0.1(0.0-0.2)$ & $13.0(9.6-22.6)$ & 0.038 & 0.039 & $<0.01$ & 0.98 & $<0.01$ & 0.57 \\
\hline SDI $(\geq 5)$ & $\%$ & $\mathrm{n} / \mathrm{a}$ & $\mathrm{n} / \mathrm{a}$ & 0.6 & $0.0(0.0-0.0)$ & $13.5(9.7-25.6)$ & 0.017 & 0.20 & 0.010 & 0.32 & 0.026 & 0.11 \\
\hline SDI $(\geq 7.5)$ & $\%$ & $\mathrm{n} / \mathrm{a}$ & $\mathrm{n} / \mathrm{a}$ & 0.1 & $0.0(0.0-0.0)$ & $5.0(3.1-10.3)$ & 0.026 & 0.18 & 0.023 & 0.21 & 0.023 & 0.20 \\
\hline
\end{tabular}

For a complete glossary of variables, see Supplementary Item 1. n/a, not applicable, because variable is not normally distributed (LR performed after log transformation).

HR, heart rate; QRS, QRS duration; QTc, rate-corrected QT interval; EMAT, electromechanical activation time; EMATc, rate-corrected electromechanical activation time; LVST, left ventricular systolic time; LVSTC, rate-corrected left ventricular systolic time; EMATc ( $\geq 15 \%)$, per cent detected $10 \mathrm{~s}$ segments in which the electromechanical activation time is greater than or equal the threshold value of $15 \%$; $S 3(\geq 5)$, per cent detected $10 \mathrm{~s}$ segments in which the power of the third heart sound is greater than or equal the threshold value of 5; $54(\geq 5)$, per cent detected $10 \mathrm{~s}$ segments in which the power of the fourth heart sound is greater than or equal the threshold value of 5; SDI ( $\geq 5)$, per cent detected $10 \mathrm{~s}$ segments in which the systolic dysfunction index is greater than or equal the threshold value of 5; SDI $\geq 7.5)$, per cent detected $10 \mathrm{~s}$ segments in which the systolic dysfunction index is greater than or equal the threshold value of 7.5 .

*Significant after Bonferroni correction $(\mathrm{P}<0.0042)$.

However, none of the diagnostic methods currently used in clinical practice can continuously record and analyse haemodynamic data characterising and quantifying systolic and diastolic ventricular function over several hours or even days. Therefore, while there is still room for improvement and development with respect to device attachment and consistent recording quality, acoustic cardiography has potential to provide added value over conventional diagnostic tools such as echocardiography, systemic blood pressure measurements or cardiac catheterisation.

Similar to other Holter ECG systems currently used in horses, automated Holter ECG analysis by Audicor ${ }^{\circledR}$ was prone to artefacts and classification error in respect to detection of APCs and VPCs, respectively. Therefore, full disclosure over-reading of the automated analysis results by a trained veterinary specialist is mandatory. The corrected Holter ECG analyses in this study indicate that atrial premature complexes were common in overnight recordings in this group of clinically healthy horses (occurring in $44.8 \%$ of all horses), whereas ventricular premature complexes were rare (occurring in $4.3 \%$ of all horses). While this finding is not unexpected and corresponds to findings reported by other authors [35-38], this is the largest original study describing Holter ECG analyses in resting, undisturbed horses. The analyses further showed that in these apparently healthy horses, up to $0.18 \%$ of analysed beats were of atrial ectopic origin and up to $0.021 \%$ of beats were of ventricular ectopic origin.

This study served to establish reference intervals for Audicor ${ }^{\circledR}$ acoustic cardiography variables in large-breed horses. The results indicate that when accounting for the family-wise statistical error, none of the measured variables, including QRS, QTC, EMAT, EMATC, LVST and LVSTC, is related to age, weight, height (Tables 1 and 2) and that all of them are reproducible, with a CV of well below $10 \%$, when measured and averaged in an overnight recording (Table 3). Day-to-day variability is considerably higher for EMAT, EMATC and LVSTC when derived from a 10-s baseline "snapshot" recording. The Audicor ${ }^{\circledR}$ variables EMAT and LVST correspond (but are not identical [21]) to the echocardiographic variables pre-ejection period (PEP) and left ventricular ejection time (LVET). Day-to-day variability in PEP (CV 6.7 and 11.9\%) and LVET (CV 6.9 and 4.5\%) measured by tissue Doppler imaging (TDI) in horses has been recently reported in the literature $[39,40]$ The respective CVs suggest that reproducibility of systolic time intervals is higher for overnight Audicor ${ }^{\circledast}$ recordings compared to single-point TDI measurements. Whether Audicor ${ }^{\circledR}$-derived EMAT and LVST will therefore be more sensitive to detect changes in LV systolic function compared to echocardiography will have to be investigated in future studies.

The normal intervals found for heart rate, QRS duration and QTc interval correspond well with those previously reported in the literature for healthy horses of similar body weight [38]. The QRS duration and consequently the $\mathrm{SDI}_{\text {bas, }}$ which is calculated as a function of QRS duration, QT interval, EMATC and S3, were significantly lower in mares compared to geldings. Because of the low number of stallions in the study group, no inference could be made regarding stallions or male horses in general. However, the literature suggests that the ORS duration in stallions exceeds those of mares by $5-10$ ms [41], a difference that is similar in magnitude to the mean difference in QRS duration of 6 ms found between mares and geldings in this study. This also corresponds to studies in people, showing that the QRS duration is significantly longer in men than in women $[42,43]$. With reference to the QT interval, it is important to note that the Bazett formula is only one of many methods for rate-correction of the QT interval, but it is neither considered the only nor the best one. In horses, it has recently been postulated that QT shortening during exercise follows a piecewise linear model [44]. However, since no modifications to the Audicor ${ }^{\circledR}$ correction methods could be made for the purpose of this study, Bazett-corrected QTC was reported.

Direct statistical comparison of the Audicor ${ }^{\circledR}$ variables EMAT and LVST with the corresponding echocardiographic variables PEP and LVET, respectively, was beyond the scope of this study. However, one would expect that EMAT, lasting from onset of the electrocardiographic $\mathrm{O}$ wave to the start of isovolumic contraction (which is timely related to the first heart sound, S1), is shorter than PEP, which lasts from onset of $Q$ to the start of ventricular ejection (corresponding to aortic valve opening and onset of aortic blood flow). Conversely, LVST, lasting from the start of isovolumic contraction (S1) to the end of ejection (which is timely related to the second heart sound, S2) should be longer than LVET, which lasts from begin to end of ventricular ejection (aortic valve opening to closure) $[21,27]$. This is concurrent with recent studies using tissue Doppler imaging for assessment of left ventricular function in horses, in which reported PEP (127 $\pm 25.3 \mathrm{~ms} ; 121 \pm 14.1 \mathrm{~ms})$ was longer than EMAT and LVET (404 \pm 18.9 ms; $421 \pm 24.7 \mathrm{~ms}$ ) was shorter than LVST reported in this 

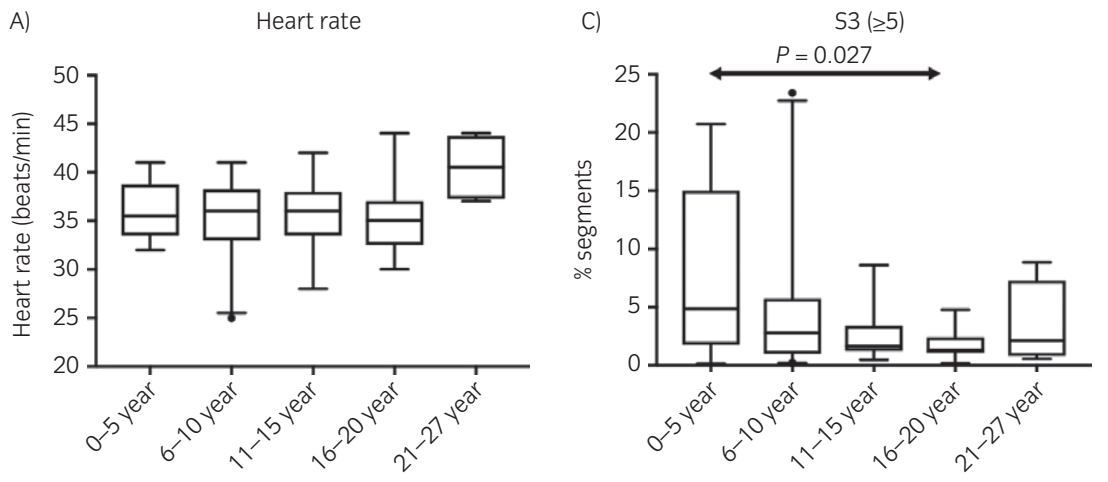

B)

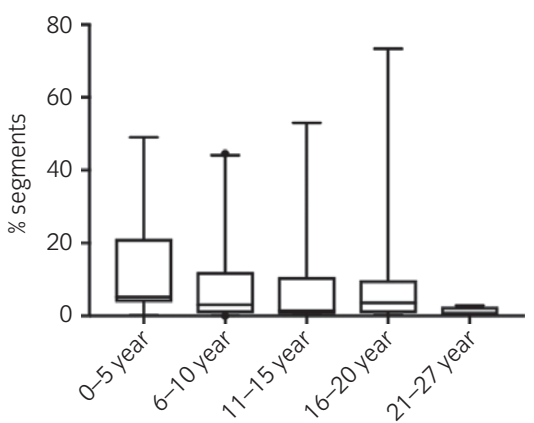

D)

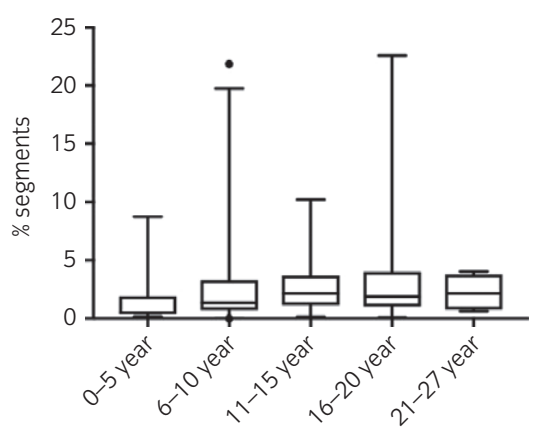

Fig 3: Comparison of heart rate, EMATc $(\geq 15 \%)$, S3 $(\geq 5)$ and S4 ( $\geq 5)$ between age groups. EMATC $(\geq 15 \%)$, per cent detected 10-s segments in which the electromechanical activation time is equal to or exceeding the threshold value of $15 \%$; $\mathrm{S3}(\geq 5)$, per cent detected 10-s segments in which the power of the third heart sound is equal to or exceeding the threshold value of 5; S4 $(\geq 5)$, per cent detected 10-s segments in which the power of the fourth heart sound is equal to or exceeding the threshold value of 5 .

TABLE 2: Summary statistics and reference intervals of baseline "snapshot" variables obtained in clinically healthy horses $(\mathrm{n}=123)$.

\begin{tabular}{|c|c|c|c|c|c|c|c|c|c|c|c|c|}
\hline \multirow[b]{3}{*}{ Variable } & \multirow[b]{3}{*}{ Unit } & \multicolumn{5}{|c|}{ Summary statistics } & \multicolumn{6}{|c|}{ Linear regression } \\
\hline & & \multirow[b]{2}{*}{ Mean } & \multirow[b]{2}{*}{ s.d. } & \multirow[b]{2}{*}{ Median } & \multirow{2}{*}{$\begin{array}{l}\text { Lower limit of } \\
\text { reference } \\
\text { interval }(90 \% \mathrm{Cl})\end{array}$} & \multirow{2}{*}{$\begin{array}{l}\text { Upper limit of } \\
\text { reference } \\
\text { interval }(90 \% \mathrm{Cl})\end{array}$} & \multicolumn{2}{|l|}{ Age } & \multicolumn{2}{|c|}{ Body weight } & \multicolumn{2}{|l|}{ Height } \\
\hline & & & & & & & $\mathrm{R}^{2}$ & $P$ value & $R^{2}$ & $P$ value & $R^{2}$ & $P$ value \\
\hline $\mathrm{HR}_{\text {bas }}$ & beats/min & 38 & 6.1 & 38 & $28(25-30)$ & $48(46-81)$ & 0.089 & $<0.001 *$ & $<0.01$ & 0.70 & $<0.01$ & 0.76 \\
\hline $\mathrm{EMAT}_{\text {bas }}$ & $\mathrm{ms}$ & 98 & 21.4 & 94 & $62(61-66)$ & 153 (137-168) & $<0.01$ & 0.57 & 0.017 & 0.15 & $<0.01$ & 0.50 \\
\hline EMATC bas & $\%$ & 5.9 & 1.8 & 6 & $3.0(3.0-4.0)$ & $9.9(9.0-16.0)$ & 0.021 & 0.11 & $<0.01$ & 0.32 & $<0.01$ & 0.46 \\
\hline LVST $_{\text {bas }}$ & ms & 492 & 43.6 & 495 & $362(310-426)$ & 563 (559-592) & 0.039 & 0.029 & $<0.01$ & 0.8 & $<0.01$ & 0.98 \\
\hline LVSTC $_{\text {bas }}$ & $\%$ & 31.2 & 4.0 & 32.0 & $22.0(20.0-25.0)$ & $38.9(38.0-41.0)$ & 0.021 & 0.11 & $<0.01$ & 0.68 & $<0.01$ & 0.76 \\
\hline $\mathrm{S} 3_{\text {bas }}$ & & 3.7 & 1.0 & 3.5 & $2.3(2.0-2.5)$ & $6.3(5.4-8.7)$ & $<0.01$ & 0.31 & 0.034 & 0.047 & $<0.01$ & 0.31 \\
\hline $\mathrm{S}_{\text {bas }}$ & & 3.1 & 1.1 & 3.1 & $1.3(0.5-1.4)$ & $5.9(4.7-7.0)$ & $<0.01$ & 0.91 & $<0.01$ & 0.85 & $<0.01$ & 0.47 \\
\hline $\mathrm{SDI}_{\text {bas }}$ & & 2.5 & 1.0 & 2.4 & $1.1(0.4-1.2)$ & $4.8(4.5-5.4)$ & 0.017 & 0.18 & $<0.01$ & 0.64 & $<0.01$ & 0.49 \\
\hline
\end{tabular}

For a complete glossary of variables, see Supplementary Item 1. HR, heart rate; EMAT, electromechanical activation time; EMATc, rate-corrected electromechanical activation time; LVST, left ventricular systolic time; LVSTc, rate-corrected left ventricular systolic time; S3, power of the third heart sound; S4, power of the fourth heart sound; SDI, systolic dysfunction index.

*Significant after Bonferroni correction $(\mathrm{P}<0.0063)$.

study, respectively $[28,39]$. Both EMAT and LVST are shorter in people compared to horses $[21,27,31]$, which can be explained by different body mass and cardiac size. Conversely, EMATc and LVSTc are higher in people compared to horses, likely related to the markedly lower resting heart rate in horses, leading to disproportionally longer diastolic time and RR interval in relation to the systolic time intervals. Linear correction of EMAT and LVST to RR is indirectly supported by findings in people, indicating that both LV PEP and LVET are linearly related to increases in heart rate [45]. To our knowledge, this has not been investigated in horses. These differences suggest that EMATc $(\geq 15 \%) \quad[20,23,30,32]$ may not be valid to predict LV systolic dysfunction in horses and that a different cut-off value will have to be established. However, these comparisons overall suggest that the concept of acoustic cardiography to continuously measure systolic time intervals is applicable to horses and provides plausible and physiologically meaningful results. Their clinical value, however, remains to be determined.

Concerning the strength of diastolic heart sounds, the data of this study indicate that both $\mathrm{S3}$ and $\mathrm{S} 4$ are detected in virtually all horses at some time point during the acoustic cardiography recordings. In people, S3 and $\mathrm{S} 4$ are considered to be present if their power is $\geq 5$ [31]. Applying this 
TABLE 3: Reproducibility of "cardiac findings" and baseline "snapshot" variables in 10 healthy horses.

\begin{tabular}{|c|c|c|c|c|c|}
\hline \multirow[b]{2}{*}{ Variable } & \multirow[b]{2}{*}{ Unit } & \multicolumn{2}{|c|}{ Summary statistics } & \multicolumn{2}{|c|}{$\begin{array}{l}\text { Between-day } \\
\text { variability }\end{array}$} \\
\hline & & $\begin{array}{l}\text { Mean } \\
\text { [Median] }\end{array}$ & $\begin{array}{l}\text { s.d. [2.5-97.5th } \\
\text { percentile] }\end{array}$ & CV (\%) & BSI \\
\hline \multicolumn{6}{|c|}{ "Cardiac findings" variables } \\
\hline $\mathrm{HR}$ & beats/min & 36 & 2.7 & 4.7 & \\
\hline QRS & $\mathrm{ms}$ & 119 & 6.1 & 0.7 & \\
\hline QTC & $\mathrm{ms}$ & 376 & 17.4 & 4.3 & 44 \\
\hline EMAT & $\mathrm{ms}$ & 105 & 9.3 & 3 & \\
\hline EMATC & $\%$ & 7.1 & 1.5 & 7.7 & 1 \\
\hline LVST & $\mathrm{ms}$ & 473 & 32.1 & 2.5 & 33 \\
\hline LVSTC & $\%$ & 28.9 & 1.1 & 2.8 & 2 \\
\hline EMATC $(\geq 15 \%)$ & $\%$ & {$[3.5]$} & {$[0.1-27.4]$} & $\mathrm{n} / \mathrm{a}$ & \\
\hline S3 $(\geq 5)$ & $\%$ & [1.7] & {$[0.4-19.7]$} & $\mathrm{n} / \mathrm{a}$ & 3. \\
\hline$S 4(\geq 5)$ & $\%$ & {$[1.4]$} & {$[0.4-8.9]$} & $\mathrm{n} / \mathrm{a}$ & 3 \\
\hline SDI $(\geq 5)$ & $\%$ & {$[0.8]$} & {$[0-7.2]$} & $\mathrm{n} / \mathrm{a}$ & 3. \\
\hline $\operatorname{SDI}(\geq 7.5)$ & $\%$ & {$[0.3]$} & {$[0-1.6]$} & $\mathrm{n} / \mathrm{a}$ & 1 \\
\hline \multicolumn{6}{|c|}{ Baseline "snapshot" variables } \\
\hline $\mathrm{HR}_{\text {bas }}$ & beats/min & 37 & 3.5 & 9.6 & 10 \\
\hline $\mathrm{EMAT}_{\text {bas }}$ & $\mathrm{ms}$ & 92 & 24.2 & 15.7 & 40.0 \\
\hline EMATC $c_{\text {bas }}$ & $\%$ & 5.2 & 1.4 & 16.4 & 2 \\
\hline LVST $_{\text {bas }}$ & $\mathrm{ms}$ & 502 & 26.9 & 3.5 & 48.2 \\
\hline LVSTC $c_{\text {bas }}$ & $\%$ & 31.1 & 2.8 & 9 & 7.7 \\
\hline $\mathrm{S}_{\text {bas }}$ & & 3.5 & 0.7 & 19.2 & 1.9 \\
\hline $54_{\text {bas }}$ & & 2.9 & 1.2 & 43.1 & 3.5 \\
\hline $\mathrm{SDI}_{\text {bas }}$ & & 2.5 & 1.3 & 34.8 & 2.4 \\
\hline
\end{tabular}

For a complete glossary of variables, see Supplementary Item 1. CV, coefficient of variation; BSI, British standards institution; HR, heart rate; QRS, QRS duration; QTc, rate-corrected QT interval; EMAT, electromechanical activation time; EMATc, rate-corrected electromechanical activation time; LVST, left ventricular systolic time; LVSTc, rate-corrected left ventricular systolic time; EMATc $(\geq 15 \%)$, per cent detected $10 \mathrm{~s}$ segments in which the electromechanical activation time is greater than or equal the threshold value of $15 \%$; S3 $(\geq 5)$, per cent detected 10 s segments in which the power of the third heart sound is greater than or equal the threshold value of 5 ; $54(\geq 5)$, per cent detected $10 \mathrm{~s}$ segments in which the power of the fourth heart sound is greater than or equal the threshold value of 5; SDI $(\geq 5)$, per cent detected $10 \mathrm{~s}$ segments in which the systolic dysfunction index is greater than or equal the threshold value of 5; SDI $(\geq 7.5)$, per cent detected $10 \mathrm{~s}$ segments in which the systolic dysfunction index is greater than or equal the threshold value of 7.5 ; S3, power (intensity and persistence) of the third heart sound, range between 0 and 10; S4, power (intensity and persistence) of the third heart sound, range between 0 and 10; SDI, systolic dysfunction index.

same cut-off, S3 was present in 0.2-19.7\% (2.5-97.5th percentile) and S4 was present in $0.1-13.0 \%$ of all 10-s segments analysed during the overnight recordings.

Prevalence of S3 is high in people less than 40 years of age and decreases thereafter, whereas prevalence of 54 increases with advanced age $[27,31,46]$. Similarly, in this study, S3 more often had a higher intensity and persistence in younger horses compared to older ones. Conversely, the fourth heart sound S4 tended to be less pronounced in young horses, but the differences were not statistically significant. These findings are consistent with earlier studies on diastolic heart sounds in horses $[29,47-$ 49]. Typically, S3 is described as a low-intensity, low-pitched early-diastolic sound that is not heard consistently in all horses but is considered a normal finding in most instances $[29,47,48]$. Occurrence of S3 is reported to be as high as 50\% $[47,48]$ and clinical experience suggests that S3 is most often heard in fit athletic animals and is a less common finding in older horses and ponies [50]. The fourth heart sound is heard in late diastole immediately prior to S1. It should be detected in nearly all horses in sinus rhythm and is particularly pronounced in horses with slow heart rate and long $\mathrm{PQ}$ interval $[5,49,50]$. In agreement with this study, the literature suggests that auscultatory characteristics of S4 appeared similar in animals of different age [49].

Intensity and persistence of diastolic heart sounds in horses can change with development of cardiac disease. Atrial fibrillation will inevitably result in an absence of S4, while the intensity of 53 can be increased in horses with severe mitral insufficiency, atrial fibrillation and heart failure [5,29,51]. In people with heart failure, increased intensity and persistence of S3 are associated with lower ejection fraction, larger left atrial and left ventricular dimensions, LV diastolic dysfunction and restrictive filling patterns $[23,30,52,53]$. The diagnostic and prognostic values of the intensity and persistence of $\mathrm{S3}$ in horses with cardiac disease are not well established. Furthermore, the description of the presence of diastolic heart sounds in horses, when assessed by auscultation, varies between clinicians and is significantly affected by training and level of experience [11]. Therefore, application of acoustic cardiography could be useful in horses with severe heart disease to objectify presence and intensity of diastolic heart sounds and to establish their clinical and prognostic value. This said, Audicor ${ }^{\circledR}$ variables characterising S3 and S4 in horses were affected by a high dayto-day variability. While it is not possible to distinguish to which extent the day-to-day variability is caused by physiological haemodynamic variation and by measurement error inherent to the technology (including variation in positioning of the microphone relative to the points of maximum intensity of S3 and S4), these findings suggest that clinically relevant alterations in $\mathrm{S} 3$ and $\mathrm{S} 4$ would need to be sufficiently large to be reliably detected in individual horses over time. Furthermore, it is unclear whether the human cut-offs of S3 $(\geq 5)$ and $S 4(\geq 5)$ can be used in horses to define the presence of diastolic heart sounds. The same is also true for the SDI, a combined index of QRS duration, QT interval, EMATc, and S3. While useful to predict LV systolic dysfunction and poor prognosis in people with heart disease $[20,23,30,32]$, day-to-day variability in SDI is high in horses. The magnitude of change seen with LV systolic dysfunction in horses and appropriate cut-off values for SDI for diagnostic and prognostic purposes are unknown. This will have to be investigated in future studies including horses with cardiac disease.

The 10-s baseline "snapshop" strip, which was derived from the first minutes of each recording, can be regarded as a rapid point-of-care test. Ideally, such a "snapshot" analysis would be sufficient to measure systolic time intervals, quantify the intensity of diastolic heart sounds and assess cardiovascular function in horses with cardiac disease in field settings, avoiding the necessity of an ambulatory long-term recording. However,

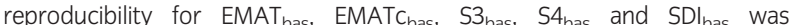
considerably lower than those for "cardiac findings" variables. Horses have a prominent resting vagal tone and a markedly lower resting heart rate compared to people, resulting in fewer and sometimes varying heart beats included in the "snapshot" analysis. Transient sympathetic stimulation immediately after instrumentation and second-degree AV blocks may also influence "snapshot" recordings to a varying degree. Hence, similar to other point-of-care diagnostic methods (e.g. manually counting heart beats for $10 \mathrm{~s}$ to calculate the heart rate in beats/min), physiologic haemodynamic variability and environmental factors likely diminish reproducibility of "snapshot" data. Potentially, reproducibility could be increased by granting an accommodation period after instrumentation and by analysing longer recordings of $20 \mathrm{~s}$ or more. However, technical restrictions in the Audicor ${ }^{\circledR}$ system make it impossible to adjust the "snapshot" time interval for use in horses so far. Therefore, the use of standard "snapshot" recordings for trending of haemodynamics over multiple days or comparing haemodynamics on different days has likely limited clinical utility in individual horses, unless the changes to be detected are sufficiently large.

The study has several limitations that should be mentioned. First, we have to acknowledge that the Audicor ${ }^{\circledR}$ algorithms were established based on human databases. While correlations of Audicor ${ }^{\circledR}$ measurements with ECG, echocardiography and invasive haemodynamics have been well established in people [32,54-58]; this was beyond the scope of this initial study. However, the 2D and 3D graphical representation of the equine phonocardiograms (Fig 1) and the reproducibility data suggest that the time-frequency analysis allowed consistent detection of all four heart sounds and sufficient definition of sound fiducial points. Also, as discussed above, comparison of Audicor ${ }^{\circledR}$ variables with published data of ECG time intervals and echocardiography-derived systolic time intervals in horses 
showed that Audicor ${ }^{\circledR}$ measurements were within sensible, physiologically meaningful ranges. Future investigations including gold standard measurements such as multilead ECG recordings, echocardiography and intracardiac pressure measurements will have to confirm this contention and will serve to better characterise the accuracy of the Audicor ${ }^{\circledR}$ method for use in horses.

Second, the study group was deemed clinically healthy based on history and physical examination, but without further examination such as echocardiography, laboratory examination or standardised exercise testing. Therefore, there is a small possibility to have included horses with some subclinical pathologies that had not become apparent during physical examination. Further studies including healthy horses and horses with cardiac disease, both verified using advanced diagnostic methods, will be required to confirm the current results and to establish appropriate cutoff values to distinguish healthy from diseased patients.

Another limitation was the loss of data because of technical difficulties with the device and the surcingle attachment. Data quality of the ECG and the phonocardiogram is largely influenced by motion artefacts and by endogenous and exogenous noises. Therefore, while it is not possible to restrain a horse for a prolonged period of time to reduce recording artefacts in long-term recordings, improvement of the surcingle mounting system will be required to ensure optimised data quality in future studies.

Even then, adjustment of the sensor position to account for the varying point of maximum intensity of the heart sounds in individual horses wil hardly be possible. However, Audicor ${ }^{\circledast}$ measurements of time intervals do not rely on absolute sound intensity but rather on an acceptable signal-tonoise ratio. As the results of this study indicate, the signal conditioning and detection models for the heart sounds were not markedly impacted by the variation in the sensor location in relation to the point of maximum intensity of the heart sounds. This can be explained by the fact that the Audicor $^{\circledR}$ method is very sensitive, so that even sound intensities that are not audible by the human ear can be reliably detected, particularly for low frequency heart sounds.

Finally, the Audicor ${ }^{\oplus}$ system only provides a single lead ECG in a baseapex-like configuration. While basic Holter ECG analyses are feasible with this setup, more advanced analyses of unusual or difficult-to-diagnose rhythm disturbances will not be possible because of the lack of additional ECG leads. Nonetheless, a single lead ECG recording is sufficient for analysis of acoustic cardiography data.

\section{Conclusion}

In conclusion, acoustic cardiography using Audicor ${ }^{\circledR}$ technology is feasible in horses. It is completely noninvasive and can easily be used in field settings. It provides single-lead ECG recordings for conventional Holter analyses including heart rate and basic rhythm diagnoses. Combination with a phonocardiogram allows continuous measurement of systolic time intervals and quantification of diastolic heart sounds over prolonged periods of time. When applied to overnight recordings, the technology provides a noninvasive method that has the potential to be used for assessment and monitoring of cardiovascular function in horses. While interpretation of ECG traces for comprehensive rhythm analyses need full disclosure over-reading by a trained veterinary specialist, calculation of acoustic cardiography variables is based on automated algorithms and therefore independent of examiner input. This study provides proof of concept for acoustic cardiography in horses and sets the stage for further studies to establish the diagnostic and prognostic value of acoustic cardiography in horses with heart disease.

\section{Authors' declaration of interests}

No competing interests have been declared.

\section{Ethical animal research}

The study was approved by the district veterinary office of the Canton of Zurich, Switzerland. Written owners' consent was obtained prior to inclusion of horses in the study.

\section{Sources of funding}

Financial support for this study was provided by the "Stiftung für Herz- und Kreislaufkrankheiten", Hergiswil, Switzerland and by the "Stiftung Pro Pferd", Zurich, Switzerland. Inovise Medical, Inc., Beaverton, OR, USA, is acknowledged for providing the Audicor ${ }^{\circledR}$ Dx Patch devices and for support with data analyses.

\section{Acknowledgements}

The authors thank all horse-owners for their collaboration.

\section{Authorship}

N. Zuber contributed to study design, study execution, data analysis and interpretation, and preparation of the manuscript. M. Zuber contributed to study design and data interpretation. C. Schwarzwald contributed to study design, data analysis and interpretation, and preparation of the manuscript. All authors gave their final approval of the manuscript.

\section{Manufacturers' addresses}

${ }^{a} A p o D x$ Technologies, Taiwan.

${ }^{b}$ Cognionics Inc., San Diego, California, USA.

Inovise Medical Inc., Beaverton, Oregon, USA.

${ }^{\mathrm{d}}$ Microsoft Corp., Redmond, Washington, USA

'GraphPad Softward Inc., San Diego, California, USA.

${ }^{f}$ National Veterinary School, Toulouse, France.

\section{References}

1. Martin, B.B. Jr, Reef, V.B., Parente, E.J. and Sage, A.D. (2000) Causes of poor performance of horses during training, racing, or showing: 348 cases (1992-1996). J. Am. Vet. Med. Ass. 216, 554-558.

2. Stevens, K.B., Marr, C.M., Horn, J.N., Pfeiffer, D.U., Perkins, J.D., Bowen, I.M., Allan, E.J., Campbell, J. and Elliott, J. (2009) Effect of left-sided valvular regurgitation on mortality and causes of death among a population of middle-aged and older horses. Vet. Rec. Case Rep. 164, 6-10.

3. Leroux, A.A., Detilleux, J., Sandersen, C.F., Borde, L., Houben, R.M., Al Haidar, A., Art, T. and Amory, H. (2013) Prevalence and risk factors for cardiac diseases in a hospital-based population of 3434 horses (19942011). J. Vet. Intern. Med. 27, 1563-1570.

4. Else, R.W. and Holmes, J.R. (1972) Cardiac pathology in the horse. 1. Gross pathology. Equine Vet. J. 4, 1-8.

5. Schwarzwald, C.C. (2018) Disorders of the cardiovascular system. In: Equine Internal Medicine, 4th edn., Eds: S.M. Reed, W.M. Bayly and D.C. Sellon, Saunders Elsevier, St. Louis, Missouri. pp 387-541.

6. Reef, V.B. (1995) Evaluation of ventricular septal defects in horses using two-dimensional and Doppler echocardiography. Equine Vet. J. 27, Suppl. 19, 86-95

7. Reef, V.B., Reimer, J.M. and Spencer, P.A. (1995) Treatment of atrial fibrillation in horses: new perspectives. J. Vet. Intern. Med. 9, 57-67.

8. Bentz, B.G., Erkert, R.S. and Blaik, M.A. (2002) Atrial fibrillation in horses: treatment and prognosis. Compendium 24, 817-822.

9. Grenacher, P.A. and Schwarzwald, C.C. (2010) Assessment of left ventricular size and function in horses using anatomical M-mode echocardiography. J. Vet. Cardiol. 12, 111-121.

10. Buhl, R., Ersboll, A.K., Eriksen, L. and Koch, J. (2004) Sources and magnitude of variation of echocardiographic measurements in normal standardbred horses. Vet. Radiol. Ultrasound. 45, 505-512.

11. Naylor, J.M., Yadernuk, L.M., Pharr, J.W. and Ashburner, J.S. (2001) An assessment of the ability of diplomates, practitioners, and students to describe and interpret recordings of heart murmurs and arrhythmia. J. Vet. Intern. Med. 15, 507-515.

12. Kriz, N.G. and Rose, R.J. (2002) Repeatability of standard transthoracic echocardiographic measurements in horses. Aust. Vet. J. 80, 362-370. 
13. Young, L.E. and Scott, G.R. (1998) Measurement of cardiac function by transthoracic echocardiography: day to day variability and repeatability in normal Thoroughbred horses. Equine Vet. J. 30, 117-122.

14. Schwarzwald, C.C., Schober, K.E. and Bonagura, J.D. (2007) Methods and reliability of echocardiographic assessment of left atrial size and mechanical function in horses. Am. J. Vet. Res. 68, 735-747.

15. Trachsel, D.S., Bitschnau, C., Waldern, N., Weishaupt, M.A. and Schwarzwald, C.C. (2010) Observer agreement for detection of cardiac arrhythmias on telemetric ECG recordings obtained at rest, during and after exercise in 10 Warmblood horses. Equine Vet J 42, Suppl. 38, 208215.

16. Erne, P. (2008) Beyond auscultation-acoustic cardiography in the diagnosis and assessment of cardiac disease. Swiss Med. Wkly 138, 439452

17. Roos, M., Toggweiler, S., Jamshidi, P., Zuber, M., Kobza, R., Meier, R. and Erne, P. (2008) Noninvasive detection of left ventricular systolic dysfunction by acoustic cardiography in cardiac failure patients. J. Card. Fail. 14, 310-319.

18. Chao, T.-F., Sung, S.-H., Cheng, H.-M., Yu, W.-C., Wang, K.-L., Huang, C.-M and Chen, C.-H. (2010) Electromechanical activation time in the prediction of discharge outcomes in patients hospitalized with acute heart failure syndrome. Inter. Med. 49, 2031-2037.

19. Zuber, M., Attenhofer Jost, C.H., Kipfer, P., Collins, S.P., Michota, F. and Peacock, W.F. (2007) Acoustic cardiography augments prolonged QRS duration for detecting left ventricular dysfunction. Ann. Noninvasive Electrocardiol. 12, 316-328.

20. Wang, S., Liu, M., Fang, F., Shang, Q., Sun, J.P., Sanderson, J.E. and Yu, C.M. (2016) Prognostic value of acoustic cardiography in patients with chronic heart failure. Int. J. Cardiol. 219, 121-126.

21. Roos, M., Toggweiler, S., Zuber, M., Jamshidi, P. and Erne, P. (2006) Acoustic cardiographic parameters and their relationship to invasive hemodynamic measurements in patients with left ventricular systolic dysfunction. Congest. Heart Fail. 12, Suppl. 1, 19-24.

22. Kobza, R., Roos, M., Toggweiler, S., Zuber, M. and Erne, P. (2008) Recorded heart sounds for identification of ventricular tachycardia. Resuscitation 79, 265-272

23. Wang, S., Fang, F., Liu, M., Lam, Y.Y., Wang, J., Shang, Q., Sun, J.P., Sanderson, J.E. and Yu, C.M. (2013) Rapid bedside identification of highrisk population in heart failure with reduced ejection fraction by acoustic cardiography. Int. J. Cardiol. 168, 1881-1886.

24. Wang, S., Lam, Y.Y., Liu, M., Fang, F., Wang, J., Shang, Q., Sun, J.P., Sanderson, J.E. and Yu, C.M. (2013) Acoustic cardiography helps to identify heart failure and its phenotypes. Int. J. Cardiol. 167, 681-686.

25. Peacock, W.F., Harrison, A. and Moffa, D. (2006) Clinical and economic benefits of using AUDICOR S3 detection for diagnosis and treatment of acute decompensated heart failure. Congest. Heart Fail. 12, Suppl. 1, 3236

26. Zuber, M., Toggweiler, S., Quinn-Tate, L., Brown, L., Amkieh, A. and Erne P. (2008) A comparison of acoustic cardiography and echocardiography for optimizing pacemaker settings in cardiac resynchronization therapy. Pacing Clin. Electrophysiol. 31, 802-811.

27. Shah, S.J. and Michaels, A.D. (2006) Hemodynamic correlates of the third heart sound and systolic time intervals. Congest. Heart Fail. 12, Suppl. 1 8-13.

28. Koenig, T.R., Mitchell, K.J. and Schwarzwald, C.C. (2017) Echocardiographic assessment of left ventricular function in healthy horses and in horses with heart disease using pulsed-wave tissue doppler imaging. J. Vet. Intern. Med. 31, 556-567.

29. Smetzer, D.L. and Smith, C.R. (1965) Diastolic heart sounds of horses. Am. Vet. Med. Ass. 146, 937-944.

30. Wen, Y.N., Lee, A.P., Fang, F., Jin, C.N. and Yu, C.M. (2014) Beyond auscultation: acoustic cardiography in clinical practice. Int. J. Cardiol. 172, 548-560.

31. Dillier, R., Zuber, M., Arand, P., Erne, S. and Erne, P. (2011) Assessment of systolic and diastolic function in asymptomatic subjects using ambulatory monitoring with acoustic cardiography. Clin. Cardiol. 34, 384-388.

32. Efstratiadis, S. and Michaels, A.D. (2008) Computerized acoustic cardiographic electromechanical activation time correlates with invasive and echocardiographic parameters of left ventricular contractility. J. Card. Fail. 14, 577-582.
33. Abbott, J.A. and Gentile-Solomon, J.M. (2017) Measurement variation and repeatability of echocardiographic variables used to estimate pulmonary artery pressure in dogs. J. Vet. Intern. Med. 31, 1622-1628.

34. Bland, M. (2000) Clinical measurement. In: An Introduction to Medical Statistics, Ed: M. Bland, Oxford University Press, New York. pp 268-293.

35. Buhl, R., Meldgaard, C. and Barbesgaard, L. (2010) Cardiac arrhythmias in clinically healthy showjumping horses. Equine Vet. J. 42, Suppl. 38, 196201

36. Barbesgaard, L., Buhl, R. and Meldgaard, C. (2010) Prevalence of exerciseassociated arrhythmias in normal performing dressage horses. Equine Vet. J. 42, Suppl. 38, 202-207.

37. Durando, M. (2010) Exercise and stress testing. In: Cardiology of the Horse, 2nd edn., Eds: C.M. Marr and I.M. Bowen, W.B. Saunders, Edinburgh. pp 139-149.

38. Schwarzwald, C.C., Kedo, M., Birkmann, K. and Hamlin, R.L. (2012) Relationship of heart rate and electrocardiographic time intervals to body mass in horses and ponies. J. Vet. Cardiol. 14, 343-350.

39. Schwarzwald, C.C., Schober, K.E. and Bonagura, J.D. (2009) Methods and reliability of tissue Doppler imaging for assessment of left ventricular radial wall motion in horses. J. Vet. Intern. Med. 23, 643-652.

40. Decloedt, A., Verheyen, T., Sys, S., De Clercq, D. and van Loon, G. (2013) Evaluation of tissue Doppler imaging for regional quantification of radial left ventricular wall motion in healthy horses. Am. J. Vet. Res. $\mathbf{7 4}$ 53-61.

41. Nielsen, K. and Vibe-Petersen, G. (1980) Relationship between QRS duration (heart score) and racing performance in trotters. Equine Vet. J. 12, 81-84.

42. Taneja, T., Mahnert, B.W., Passman, R., Goldberger, J. and Kadish, A. (2001) Effects of sex and age on electrocardiographic and cardiac electrophysiological properties in adults. Pacing Clin. Electrophysiol. 24, 16-21.

43. Simonson, E., Blackburn, H., Puchner, T., Eisenberg, P., Ribeiro, F. and Meja, M. (1960) Sex differences in the electrocardiogram. Circ. J. 22, 598601

44. Pedersen, P.J., Kanters, J.K., Buhl, R. and Klaerke, D.A. (2013) Normal electrocardiographic OT interval in race-fit Standardbred horses at rest and its rate dependence during exercise. J. Vet. Cardiol. 15, 23-31.

45. Mertens, H.M., Mannebach, H., Trieb, G. and Gleichmann, U. (1981) Influence of heart rate on systolic time intervals: effects of atrial pacing versus dynamic exercise. Clin Cardiol. 4, 22-27.

46. Collins, S.P., Arand, P., Lindsell, C.J., Peacock, W.F.T. and Storrow, A.B. (2005) Prevalence of the third and fourth heart sound in asymptomatic adults. Congest. Heart Fail. 11, 242-247.

47. Smith, C.R., Smetzer, D.L., Hamlin, R.L. and Watsabaugh, C. (1962) Normal heart sounds and heart murmurs in the horse. Proc. Am. Ass. Equine Practnrs. 8, 49-64.

48. Patterson, D.F., Detweiler, D.K. and Glendenning, S.A. (1965) Heart sounds and murmurs of the normal horse. Ann. N. Y. Acad. Sci. 127, 242-305.

49. Smetzer, D.L., Smith, C.R. and Hamlin, R.L. (1965) The fourth heart sound in the equine. Ann. N. Y. Acad. Sci. 127, 306-321.

50. Patteson, M.W. (1996) Clinical examination. In: Equine Cardiology, Ed: M.W. Patteson, Blackwell Science Ltd, Oxford. pp 41-69.

51. Reef, V.B., Bain, F. and Spencer, P.A. (1998) Severe mitral regurgitation in horses: clinical, echocardiographic and pathological findings. Equine Vet. J. 30, 18-27.

52. Shapiro, M. Moyers, B. Marcus, G.M., Gerber, I.L., McKeown, B.H., Vessey, J.C., Jordan, M.V., Huddleston, M., Foster, E., Chatterjee, K. and Michaels, A.D. (2007) Diagnostic characteristics of combining phonocardiographic third heart sound and systolic time intervals for the prediction of left ventricular dysfunction. J. Card. Fail. 13, 18-24.

53. Zuber, M., Kipfer, P. and Attenhofer Jost, C. (2006) Systolic dysfunction: correlation of acoustic cardiography with Doppler echocardiography. Congest. Heart Fail. 12, Suppl. 1, 14-18.

54. Shah, S.J., Nakamura, K., Marcus, G.M., Gerber, I.L., McKeown, B.H., Jordan, M.V., Huddleston, M., Foster, E. and Michaels, A.D. (2008) Association of the fourth heart sound with increased left ventricular enddiastolic stiffness. J. Card. Fail. 14, 431-436.

55. Lee, E., Drew, B.J., Selvester, R.H. and Michaels, A.D. (2009) Sequence of electrocardiographic and acoustic cardiographic changes and angina during coronary occlusion and reperfusion in patients undergoing 
percutaneous coronary intervention. Ann. Noninvasive Electrocardiol. 14, 137-146

56. Karebsheh, S. and Michaels, A.D. (2011) Acoustic cardiographic indices of transmyocardial ischemia during percutaneous coronary intervention. Acute Card. Care 13, 3-8.

57. Gupta, S., Gupta, N., Arand, P. and Michaels, A.D. (2011) Computerized acoustic cardiography correlates with echocardiography and invasive haemodynamics after percutaneous transvenous mitral commissurotomy. J. Med. Eng. 35, 59-64.

58. Marcus, G.M., Gerber, I.L., McKeown, B.H. Vessey, J.C Jordan, M.V., Huddleston, M., McCulloch, C.E., Foster, E., Chatterjee, K. and Michaels, A.D. (2005) Association between phonocardiographic third and fourth heart sounds and objective measures of left ventricular function. JAMA 293, 2238-2244

\section{Supporting Information}

Additional Supporting Information may be found in the online version of this article at the publisher's website:

Supplementary Item 1: Glossary of measured variables. Supplementary Item 2: Findings by age. 


\section{Curriculum Vitae}

Vorname Name

Geburtsdatum

Geburtsort

Nationalität

Heimatort bei Schweizern

Aug. 1997 - Juli 2002:

Aug. 2002 - Juli 2006:

Aug. 2006 - Juli 2010:

2010

Sept. 2010 - Juli 2013:

Sept. 2013 - Dez. 2015:

Dez. 2015

Sept. 2016 - Nov. 2017
Aug. 2018

Sept. 2018 - Offen
Nicole Zuber

12.06.1990

Brugg AG

Schweizerin

Othmarsingen AG, Matzendorf SO

Primarschule (Othmarsingen, Schweiz)

Oberstufe (Bezirksschule, Lenzburg, Schweiz)

Kantonsschule (Kantonsschule Aarau, Aarau, Schweiz)

Matura (Alte Kantonsschule Aarau, Aarau, Schweiz)

Bachelor of Veterinary Medicine (Universität Bern, Bern, Schweiz)

Master of Veterinary Medicine (Universität Bern, Bern, Schweiz)

Abschlussprüfung vet. med. (Universität Bern, Bern, Schweiz)

Anfertigung der Dissertation

unter Leitung von Prof. Dr. med. vet. Colin C. Schwarzwald

am Departement für Pferde

der Vetsuisse-Fakultät Universität Zürich

Direktor Prof. Dr. med. vet. Colin C. Schwarzwald

Assistenzärztin in der Kleintierklinik BolligerTschuor, Oftringen, Schweiz

Assistenzärztin in der Trivet Praxis, Frick, Schweiz 